Article

\title{
Fosfomycin Resistance Evolutionary Pathways of Stenotrophomonas maltophilia in Different Growing Conditions
}

\author{
Teresa Gil-Gil $^{1,2}$ (D) and José L. Martínez ${ }^{2, *(D)}$ \\ 1 Programa de Doctorado en Biociencias Moleculares, Universidad Autónoma de Madrid, 28049 Madrid, Spain; \\ tgil@cnb.csic.es \\ 2 Centro Nacional de Biotecnología, CSIC, Darwin 3, 28049 Madrid, Spain \\ * Correspondence: jlmtnez@cnb.csic.es
}

Citation: Gil-Gil, T.; Martínez, J.L. Fosfomycin Resistance Evolutionary Pathways of Stenotrophomonas maltophilia in Different Growing Conditions. Int. J. Mol. Sci. 2022, 23, 1132. https://doi.org/ 10.3390/ijms23031132

Academic Editor: Sergei A

Subbotin

Received: 16 December 2021

Accepted: 14 January 2022

Published: 20 January 2022

Publisher's Note: MDPI stays neutral with regard to jurisdictional claims in published maps and institutional affiliations.

Copyright: (C) 2022 by the authors. Licensee MDPI, Basel, Switzerland. This article is an open access article distributed under the terms and conditions of the Creative Commons Attribution (CC BY) license (https:// creativecommons.org/licenses/by/ $4.0 /)$.

\begin{abstract}
The rise of multidrug-resistant Gram-negative pathogens and the lack of novel antibiotics to address this problem has led to the rescue of old antibiotics without a relevant use, such as fosfomycin. Stenotrophomonas maltophilia is a Gram-negative, non-fermenter opportunistic pathogen that presents a characteristic low susceptibility to several antibiotics of common use. Previous work has shown that while the so-far described mechanisms of fosfomycin resistance in most bacteria consist of the inactivation of the target or the transporters of this antibiotic, as well as the production of antibiotic-inactivating enzymes, these mechanisms are not selected in S. maltophilia fosfomycinresistant mutants. In this microorganism, fosfomycin resistance is caused by the inactivation of enzymes belonging to its central carbon metabolism, hence linking metabolism with antibiotic resistance. Consequently, it is relevant to determine how different growing conditions, including urine and synthetic sputum medium that resemble infection, could impact the evolutionary pathways towards fosfomycin resistance in S. maltophilia. Our results show that S. maltophilia is able to acquire high-level fosfomycin resistance under all tested conditions. However, although some of the genetic changes leading to resistance are common, there are specific mutations that are selected under each of the tested conditions. These results indicate that the pathways of S. maltophilia evolution can vary depending on the infection point and provide information for understanding in more detail the routes of fosfomycin resistance evolution in S. maltophilia.
\end{abstract}

Keywords: Stenotrophomonas maltophilia; experimental evolution; fosfomycin resistance

\section{Introduction}

Antibiotic resistance is nowadays one of the main problems for public health [1], and regarding this threat, the dissemination of multidrug-resistant Gram-negative bacteria is of great concern. One of these Gram-negative pathogens is Stenotrophomonas maltophilia, an opportunistic nosocomial pathogen causing different infections and characterized by its low intrinsic susceptibility to broad-range antibiotics [2]. Infections associated with S. maltophilia include serious nosocomial infections such as respiratory tract infections, bacteremia, biliary sepsis, bones, joints, soft tissues, or eye infections, endophthalmitis, dacryocystitis, endocarditis, meningitis and urinary tract infections (UTIs) [3,4]. However, UTIs caused by this microorganism have been rarely reported [5]. Although S. maltophilia can acquire antibiotic resistance genes through horizontal gene transfer (HGT) [6], mutation is the main cause for the emergence of antibiotic resistance by this organism, mainly when causing chronic infections in cystic fibrosis patients [7-9].

The increased resistance of Gram-negative bacteria to currently used antibiotics, together with the lack of new antibiotics, has renewed the interest in old less-used antibiotics such as fosfomycin [2], which is being used as a first-line treatment for the treatment of UTIs and has been proposed for treating other infections [10,11]. Fosfomycin binds to MurA 
(UDP-N-acetylglucosamine enolpyruvyl transferase), which catalyzes the first step in peptidoglycan biosynthesis and inactivates it, leading to bacterial cell lysis [12]. Although $S$. maltophilia is considered intrinsically resistant to fosfomycin, previous studies have shown that current methods for measuring fosfomycin susceptibility, which include glucose-6phosphate in their formulation, are not accurate enough for this microorganism (Gil-Gil and Martinez, submitted). Further, it has been shown that the phenotype of antibiotic resistance may depend on the environment—and hence on the infection point [13] — as well as on the conditions of selection [14]. Consequently, it is of relevance to search the mechanisms of fosfomycin resistance that $S$. maltophilia can acquire under different conditions, including urine and synthetic cystic fibrosis sputum medium (SCFM), which are representative of infective conditions.

It was previously shown that the main causes of acquiring fosfomycin resistance in different bacteria are mutations in the genes encoding the fosfomycin target MurA [15] or its transporters, UhpT (glucose-6-phosphate transporter) and GlpT (glycerol-3-phosphate transporter) [16,17], as well as the activity of fosfomycin-inactivating enzymes such as the glutathione transferase FosA [18]. However, none of these mechanisms have been previously reported to be involved in the acquisition of increasing levels of fosfomycin resistance in single-step S. maltophilia mutants. In this microorganism, mutations in genes encoding enzymes of the central metabolism are on the basis of fosfomycin acquired resistance; in particular, mutations that inactivate glycolytic enzymes belonging to the EmbdenMeyerhof-Parnas (EMP) metabolic pathway [19] are responsible for the acquisition of resistance. This indicates that $S$. maltophilia fosfomycin resistance is linked to the bacterial metabolism, a feature also supported by recent data showing that changes in the transcriptome of S. maltophilia when challenged by either fosfomycin, phosphoenolpyruvate (PEP) or glyceraldehyde-3-phosphate are very similar [20]. Given the observed close relationship between fosfomycin resistance and S. maltophilia metabolism, we challenged S. maltophilia on experimental evolution studies with increasing concentrations of fosfomycin in different media- LB, urine and SCFM — and analyzed the mutations arising after each evolution. These evolutions allowed the determination of genetic changes underlying fosfomycin antibiotic resistance in different conditions, including laboratory growing conditions and situations that mimic those that S. maltophilia find when producing urinary or lung infections. Our results reinforce the concept that metabolic changes can alter the patterns and phenotypes of evolution towards antibiotic resistance [21].

\section{Results}

\subsection{Experimental Evolution upon Fosfomycin Challenge Leads to High Levels of Resistance in} S. maltophilia Populations under all Tested Conditions

In order to elucidate how growing conditions may impact the acquisition of fosfomycin resistance by $S$. maltophilia, four independent $S$. maltophilia $\mathrm{D} 457$ populations were serially passaged for $72 \mathrm{~h}$ in the presence of increasing concentrations of fosfomycin in LB, urine or SCFM until a concentration of 4 minimum inhibitory concentration (MIC) was reached. In parallel, four independent control replicates were also passaged under the same conditions in the absence of antibiotics. It is worth mentioning that $S$. maltophilia growth increased urine $\mathrm{pH}$ from 7 to 8 .

Afterwards, MICs for the fosfomycin-evolved populations, as well as the wild-type strain D457 and the control-evolved populations, were determined by double dilution in MH, LB, urine and SCFM. As shown in Table 1, all populations exhibited high-level resistance to fosfomycin in comparison with the parental strain D457 and the control-evolved populations, reaching at least MIC increases of 16-fold compared with the parental strain. In addition, fosfomycin-evolved populations show a fosfomycin resistance phenotype in all tested media, both in those where they have evolved and in those where they did not. These results suggest that the acquired mutations are able to cause resistance in all tested media, no matter in which medium they have been selected. 
Table 1. Fosfomycin MICs of the S. maltophilia evolved populations. Fosfomycin susceptibility was determined by double dilution in populations after $72 \mathrm{~h}$ of experimental evolution in the presence of increasing concentrations of fosfomycin.

\begin{tabular}{ccccc}
\hline \multirow{2}{*}{ Population } & \multicolumn{4}{c}{ MIC $(\mu \mathrm{g} / \mathbf{m L})$} \\
\cline { 2 - 5 } & MH & LB & Urine & SCFM \\
\hline D457 & 256 & 128 & 256 & 512 \\
LB control & 256 & 128 & 128 & 256 \\
LB A & 16,384 & $>16,384$ & 6250 & $>16,384$ \\
LB B & 16,384 & $>16,384$ & 6250 & $>16,384$ \\
LB C & 16,384 & 8192 & 3125 & $>16,384$ \\
LB D & 16,384 & 5892 & 3125 & $>16,384$ \\
Urine control & 256 & 128 & 128 & 256 \\
Urine A & $>16,384$ & $>16,384$ & $>16,384$ & 16,384 \\
Urine B & $>16,384$ & $>16,384$ & $>16,384$ & $>16,384$ \\
Urine C & $>16,384$ & $>16,384$ & $>16,384$ & $>16,384$ \\
Urine D & $>16,384$ & $>16,384$ & $>16,384$ & $>16,384$ \\
SCFM control & 256 & 128 & 128 & 256 \\
SCFM A & $>16,384$ & 8192 & 6250 & $>16,384$ \\
SCFM B & 8192 & 1024 & 3125 & 8192 \\
SCFM C & 8192 & 8192 & 6250 & $>16,384$ \\
SCFM D & 16,384 & 1024 & 1562 & 4092 \\
\hline
\end{tabular}

\subsection{Selected Mutations in the Presence of Fosfomycin}

Whole-genomic DNA of evolved populations in the presence of fosfomycin and of the controls, grown in the absence of the antibiotic, as well as the DNA of the parental strain were extracted and sequenced, with the aim of determining the genetic changes potentially responsible for the decreased susceptibility to fosfomycin. Only those mutations that were selected upon antibiotic selective pressure but were absent in the populations evolved in the absence of selection were taken into consideration and are listed in Table 2. In order to confirm the presence of the genetic changes identified by whole-genome sequencing (WGS), the sequences containing the different mutations were amplified and Sanger-sequenced from stored samples of each population. Altogether, genetic modifications were found in different genes, single-nucleotide polymorphisms (SNPs) causing amino acid substitutions being the most common changes.

The four populations that evolved in LB in the presence of fosfomycin shared a deleterious mutation in eno, which encodes a phosphopyruvate hydratase (Eno), with a prevalence of $93-98 \%$ within the analyzed populations. The finding of this SNP in the eno gene reinforces previous results showing that the mutational inactivation of different enzymes belonging to the EMP central metabolism pathway, including Eno, is on the basis of fosfomycin resistance acquisition in S. maltophilia [19]. In addition, in population B a genetic change was observed in phaR, encoding a polyhydroxyalkanoate synthesis repressor, even though the percentage of reads that contain the variation within the heterogeneous population is only $22 \%$. 
Table 2. WGS-identified mutations in the fosfomycin-evolved lineages.

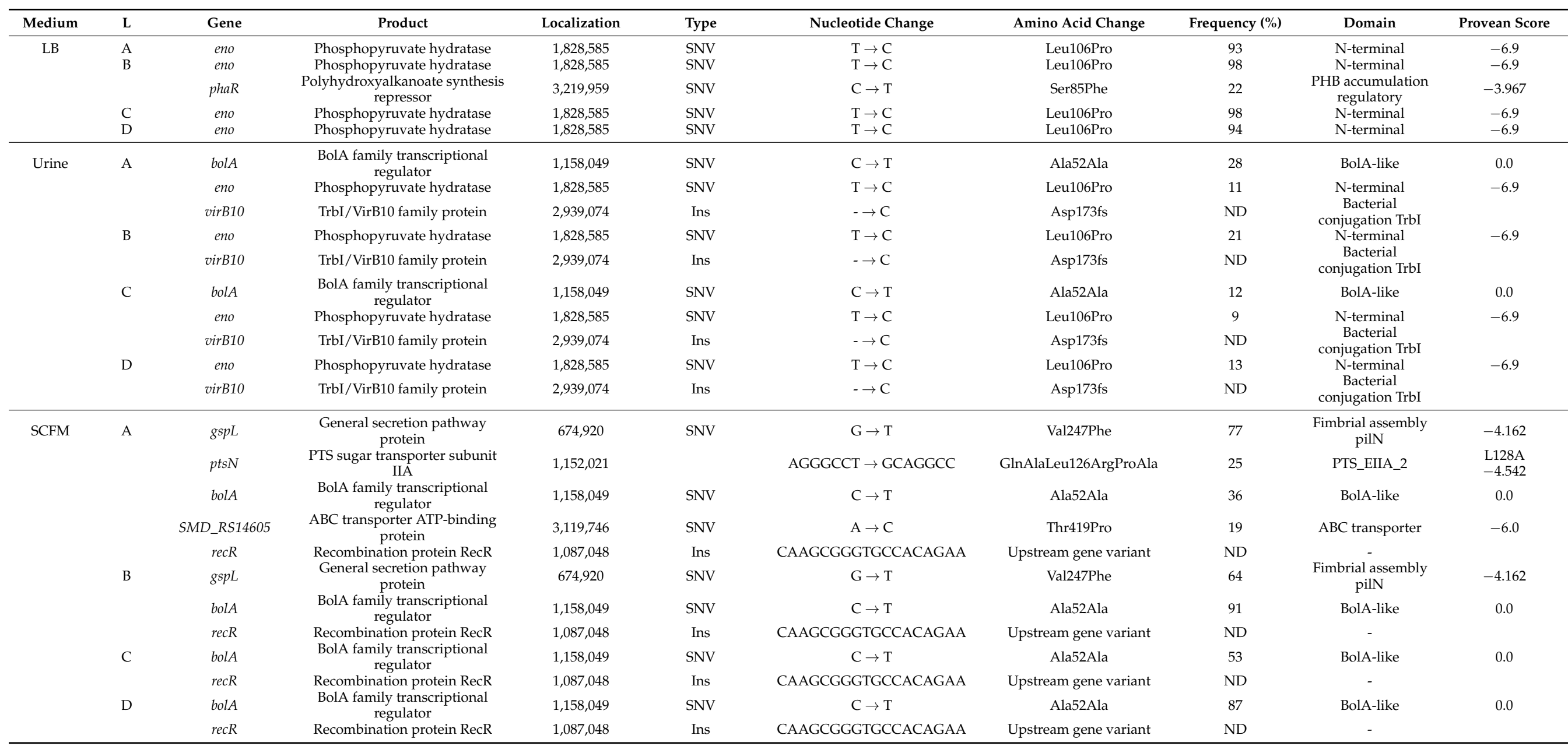

L: lineage; SNV: single-nucleotide variant; Ins: insertion; fs: frame shift; frequency (\%): percentage of reads that contain the variation within a heterogeneous population; ND: non-determined (IGV 2.11.9 Genomics Software does not determine the frequency of insertion/deletions). PROVEN score: default threshold is -2.5 , that is, variants with a score equal to or below -25 are considered "deleterious", and variants with a score above -2.5 are considered "neutral". 
Moving to urine-evolved populations, all of them share two genetic changes. Firstly, the four of them present the same mutation in eno that has been previously found in the LB evolution. Nevertheless, the frequency of this mutation drops to $9-21 \%$ in the case of urine-evolved populations; this fact could explain the presence of extra mutations found in this medium unlike LB to reach the same level of resistance. Secondly, a one-base insertion in virB10 (SMD_RS13785) was detected in the four populations. VirB10 is one of the proteins forming the type IV secretion system (T4SS) outer membrane channel, which traverses both the inner and outer membrane, playing an important role in the regulation of substrate transfer to the extracellular space [22]. Mutations in this gene have been previously linked to enhanced susceptibility to vancomycin [23]. This may suggest that a possible change in the outer membrane permeability due to this mutation allows bidirectional movement. substrate release to cell surface and uptake into the periplasm, of molecules too large to diffuse through porins across the outer membrane. To ascertain if this mutation alters the activity of vancomycin as described, the susceptibility of the fosfomycinevolved populations to this antibiotic was determined. No changes were observed with respect to the parental strain or the evolved strains in the absence of antibiotic, all of them presenting a vancomycin MIC of $256 \mu \mathrm{g} / \mathrm{mL}$. Last, an SNP in SMD_RS05475 encoding a BolA family transcriptional regulator in populations $\mathrm{A}$ and $\mathrm{C}$ was also detected. Even though this mutation does not produce an amino acid change and is considered neutral, its genomic location, in the same operon and upstream murA, the only known fosfomycin target, might suggest that this SNP could affect murA expression (see below).

Genetic modifications in five different genes were found in SCFM-evolved populations, all of them sharing the same mutation in bolA previously found in urine-evolved populations and an $18 \mathrm{bp}$ insertion that results in a genomic variant upstream of recR. In addition to these common changes, population A and B show an SNP in $g s p L$, which encodes a PilN domain-containing protein which takes part in the type II secretion system (T2SS). T2SS of S. maltophilia promotes bacterial virulence and hence human infection, but has not been previously related to antibiotic resistance [24]. Aside from previously detailed changes, population A presents two extra SNPs. The first of them is located in SMD_RS14605, encoding a protein that is part of an ABC transporter that shares a $97.83 \%$ identity with lipid A export ATP-binding/permease inner membrane protein MsbA of Pseudomonas aeruginosa. This protein is involved in lipid A transport across the cytoplasmic membrane from the inner to the outer leaflet of the inner membrane [25-29]; its deficiency has been associated with hypersensitivity to glutaraldehyde, erythromycin and hydrophobic antibiotics, such as rifampicin, in Helicobacter pylori and Lactobacillus lactis [30,31]. The last change in population A was found in $p t s N$, a nitrogen regulatory protein paralogous to the enzyme IIA ${ }^{\text {fru }}$ of the PEP-dependent carbohydrate phosphotransferase system (PTS) [32,33]. PtsN is also known as Enzyme IIA ${ }^{\mathrm{Ntr}}$, taking part in a phosphorelay system $\left(\mathrm{PTS}^{\mathrm{Ntr}}\right)$ in Escherichia coli that may have a role in the regulation of nitrogen metabolism [34,35] and that regulates the activity and expression of potassium transporters [36-38].

\subsection{Antibiotic Susceptibility Profile of the Fosfomycin-Evolved Populations}

To explore the influence of mutations selected under fosfomycin pressure on crossresistance and/or collateral sensitivity to other antibiotics, MICs of antibiotics belonging to different classes were measured at the end of each evolution (Figure 1).

In all analyzed conditions, some fosfomycin-evolved populations with a decreased susceptibility to colistin-LB-evolved populations A, B and C; urine B and D; and SCFM $\mathrm{A}$ and $\mathrm{B}$ - and polymyxin $\mathrm{B}$ - urine-evolved populations $\mathrm{C}$ and $\mathrm{D}$-were found. Further, low susceptibility to ceftazidime was observed in the urine-evolved $C$ population. The increased resistance to colistin observed in most populations may be associated with a change in permeability, as this antibiotic interacts electrostatically with the outer membrane of Gram-negative bacteria, displaces divalent cations which stabilize the lipopolysaccharide (LPS) layer and thus disrupts the membrane integrity and diffuses through it inside the cell [39]. 
A

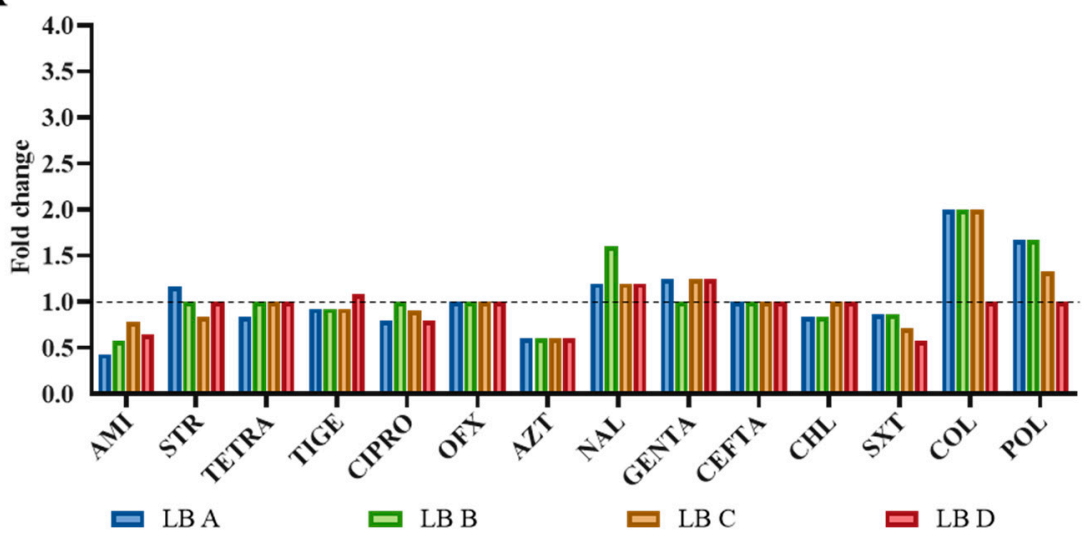

B

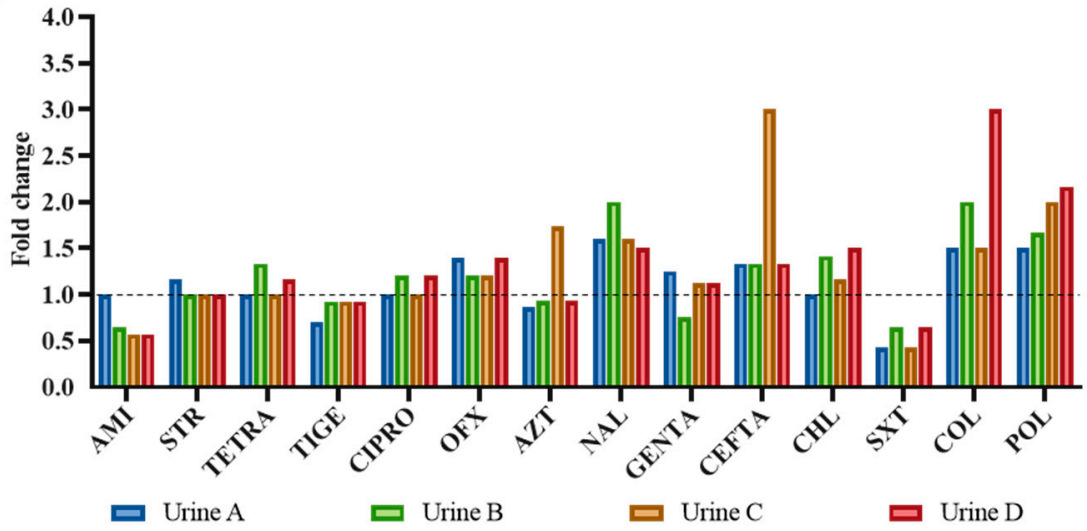

C

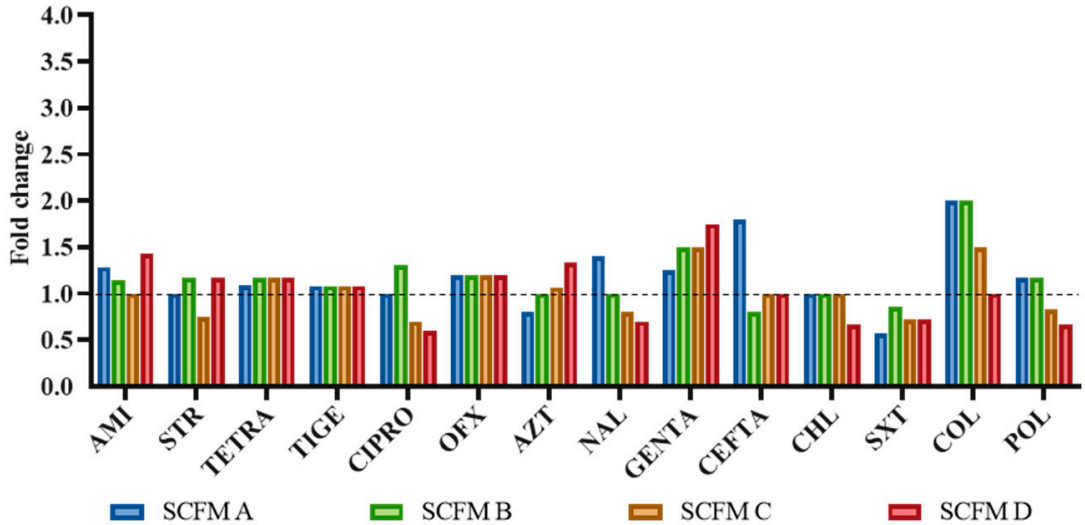

Figure 1. Collateral susceptibility of the S. maltophilia evolved populations to antibiotics from different families. MICs were determined in the twelve fosfomycin-evolved populations using the MIC values of the wild-type strain D457 as reference (dashed line). (A) LB-evolved populations; (B) urine-evolved populations; (C) SCFM-evolved populations. Values of at least double or half the wild-type MIC were considered significant. AMI, amikacin; STR, streptomycin; TETRA, tetracycline; TIGE, tigecycline; CIPRO, ciprofloxacin; OFX, ofloxacin; AZT, aztreonam; NAL, nalidixic acid; GENTA, gentamicin; CEFTA, ceftazidime; CHL, chloramphenicol; SXT, trimethoprim/sulfamethoxazole; COL, colistin; POL, polymyxin B.

Regarding collated sensitivity, susceptibility to some antibiotics was detected. Population LB A presented collateral susceptibility to amikacin, and urine A and C to trimethoprim/sulfamethoxazole (SXT), which is of relevance since this antibiotics combination is the first-line treatment for S. maltophilia infections. Similar increased SXT susceptibility 
is observed, even if it is not of significant value, in all fosfomycin-evolved populations, suggesting that the administration of fosfomycin together with SXT for the treatment of S. maltophilia infections might be taken into consideration.

\subsection{Fitness Costs Associated with Fosfomycin Resistance}

The relative fitness costs to the 12 fosfomycin-evolved populations were assessed by measuring their growth rates and comparing them with that of the D457 wild-type strain in each medium. Firstly, in LB laboratory medium, LB-evolved populations showed around 11 to $20 \%$ deficiency in their growth rate, 17 to $44 \%$ in urine populations and around 5 to $7 \%$ deficiency in SCFM evolutions (Figure 2A). On the one hand, these results indicate that LB-evolved populations present a better growth rate than those that have evolved in urine, although SCFM populations present a better growth rate, indicating that populations presenting mutations in genes encoding enzymes of the central metabolism have a greater relative fitness cost. On the other hand, virB10 mutations could be the cause of the differences in fitness cost between urine and LB-evolved populations. Secondly, when tested in urine, LB-evolved populations had a growth deficiency from 10 to $14 \%$ and SCFM-evolved populations present values ranging from 6 to $13 \%$. However, urine-evolved populations were not defective in their growth. Notably, they presented a greater growth rate than the D457 wild-type strain (Figure 2B); suggesting that selected mutations in this medium, apart from increasing resistance to fosfomycin, allow a better growth in the selection medium. It is then possible that the one-pair insertion in virB10 gene would be selected for improving the growth of S. maltophilia in urine. Thirdly, the growth in SCFM of all the evolved populations shows a deficiency with respect to the parental strain, reaching from 52 to $65 \%$ in the populations evolved in LB, 14 to $39 \%$ in the case of those evolved in urine-whose differences do not seem to be related to the selected mutations in each of them-and between 22 and 34\% in those evolved in SCFM (Figure 2C). Taken together, these results show that the selection of mutations in each evolution is occasionally-as in the case of urine- but not always related to a growth improvement in the medium in which the experimental evolution takes place.

\subsection{Expression Changes in Possible Resistance Determinants in the Fosfomycin-Evolved Populations}

Apart from the mutation found in the eno gene described as a fosfomycin resistance mechanism in our bacterium of interest, most of the genetic changes detected by WGS did not show a direct correlation with fosfomycin resistance mechanisms previously described for other bacterial species. To ascertain if these mutations produce changes in the expression level of these genes, their expression was analyzed by quantitative reverse-transcription PCR (qRT-PCR) (Figure 3). As mentioned, bolA is transcribed in an operon with murA gene. It has been previously assessed that an increased expression of bolA occurs under cell envelope stress conditions and its deletion produce changes in the outer membrane, making it more permeable and increasing susceptibility to vancomycin and fosfomycin [40,41]. However, a significant change in the expression of bolA was not observed; neither in the populations that present the mutation in that gene nor in any other population (Figure 3A). Despite the lack of changes in the expression of this gene, and since mutations were located upstream of the gene encoding the fosfomycin target murA, the expression of this gene was measured. Our results show that fosfomycin-evolved populations with a mutation in bolA gene exhibit greater murA expression (Figure 3B). Hence, the enhanced expression of this gene may be the main reason for the fosfomycin resistance of these fosfomycin-evolved populations, since an increased synthesis of this enzyme confers a resistant phenotype [42]. To further confirm this issue, MurA activity was also measured in all evolved populations. Consistent with the results dealing with murA expression, we observed that those populations with a mutation in bolA presented increased MurA activity compared with the wild-type strain (Figure 4). These results confirm that murA overexpression is on the basis of the resistance phenotype of these populations. 
$\mathbf{A}$

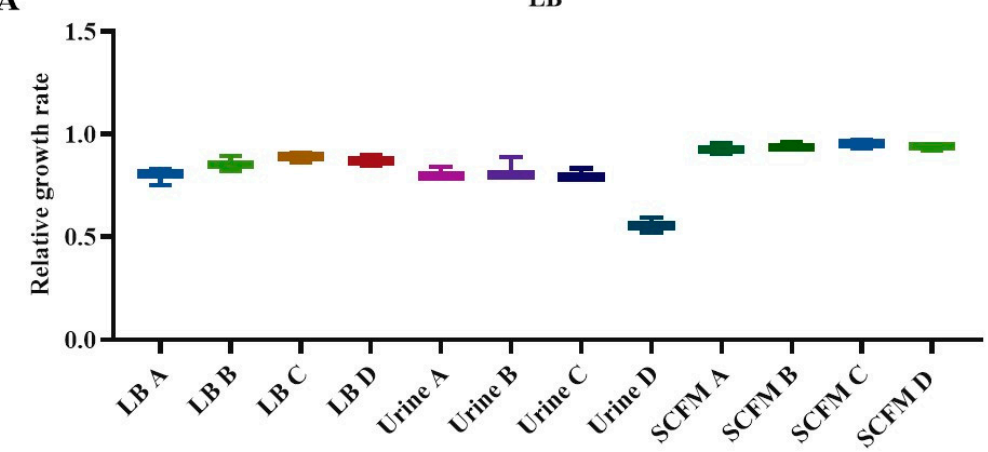

B

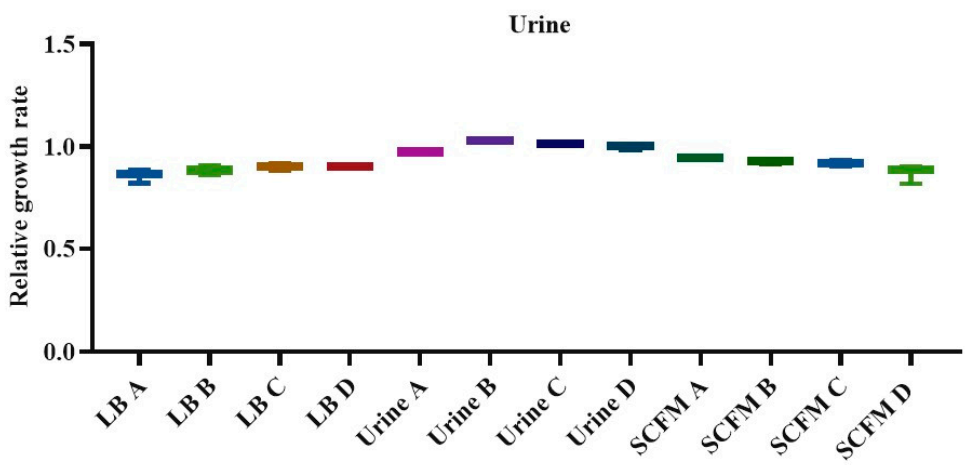

C

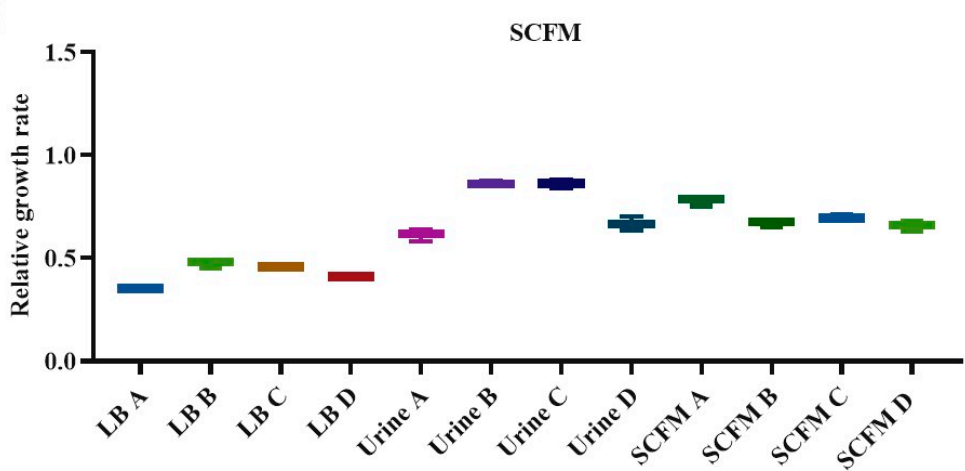

Figure 2. Fitness costs of the fosfomycin-evolved S. maltophilia populations. Growth experiments were performed in the four evolved populations. Growth rates were calculated from $\mathrm{OD}_{600}$ values corresponding to exponential growth. Relative growth rates were calculated using the wild-type D457 value as a reference. Growth was measured in LB (A), urine (B) and SCFM (C) mediums. Error bars represent the standard deviation from three independent replicates.

Regarding virB10, urine A and D fosfomycin-evolved populations showed an increased expression of this gene (Figure 3C). VirB10-defective mutants have been previously proved to exhibit enhanced sensitivity to molecules that do not normally cross the outer membrane through porins, such as vancomycin [23]. Notwithstanding, as previously mentioned, these populations do not present changes in the susceptibility to this antibiotic. All the same, since the fosfomycin gates of $S$. maltophilia are not known, it is still possible that mutations and/or changes in the expression of the T4SS would be related to fosfomycin resistance, besides its role in virulence.

A common WGS change found in all SCFM-evolved populations is an $18 \mathrm{pb}$ insertion located upstream of $r e c R$, encoding a protein involved in DNA recombination. In order to determine if this change produces a direct effect on $r e c R$, the expression of this gene was measured. However, no changes were observed in its level of expression (Figure 3D). Finally, no changes were observed in the levels of expression of $g s p L$, SMD_RS14605 or ptsN 
in any of the SCFM-evolved populations, including the SCFM linages A and B in which mutations in these genes were found (Figure $3 \mathrm{E}-\mathrm{G}$ ).

2.6. Activity of Central Metabolism Enzymes Is Associated with Fosfomycin Resistance in Some of the Fosfomycin-Evolved Populations

The only mechanism of acquired resistance to fosfomycin described to date in S. maltophilia is the impaired activity of enzymes of the lower glycolysis pathway [19]. Our results suggest that some of the fosfomycin-evolved populations could share this resistance mechanism. To determine whether the lower glycolytic pathway is involved in the fosfomycin resistance of the fosfomycin-evolved populations, the enzymatic activity of glyceraldehyde-3-phosphate dehydrogenase (Gap), phosphoglycerate kinase (Pgk), phosphoglycerate mutase (Gpm), Eno and pyruvate kinase (Pyk) was measured in the evolved-populations and in the wild-type strain. Moreover, the activity of the main dehydrogenase glucose-6-phosphate dehydrogenase (Zwf) was measured to determine the general physiological state of the populations.

As shown in Figure 5, in LB-evolved populations, Gap, Eno and Pyk activities decrease in all four populations in relation to the parental strain (Figure $5 A, D, E$ ). Thus, Eno mutation causes a loss of function of the encoded protein and a metabolic rearrangement that modifies the mutated pathway. Urine-evolved populations share the same eno mutation with LB-evolved populations, although no changes were observed in the activity of this enzyme (Figure 5D). The low proportion of this mutation in the study populations could be the cause of the lack of change in Eno activity. To ascertain if this was the reason for the observed results, individual clones from each urine-evolved population were isolated on LB agar plates and those presenting the eno mutation were chosen for further analysis. The selected clones had a resistance value to fosfomycin identical to that of the population they were selected from. As shown in Figure 6, isolated clones presented defects in eno activity in comparison with the wild-type strain. This last result shows that the reason why a deficiency in the activity of eno was not found in the urine-evolved populations was indeed the low proportion of this mutation in such populations. In addition, Pgk activity decreased in urine $C$ and $D$ fosfomycin-evolved populations (Figure 5B). In addition, Zwf activity, which is a key element in the bacterial oxidative response [43], increased with a significant change in all urine-evolved populations compared with the wild-type D457 strain (Figure 5F). These results suggest that urine-evolved populations show a different physiological state than the wild-type strain.

Finally, the resistance mechanisms of SCFM-evolved populations do not seem to be related to a metabolic shift, showing the same general physiological state as the parental D457 strain (Figure 5).

All in all, both single-step S. maltophilia fosfomycin-resistant mutants [19] and fosfomycinevolved populations, in both cases obtained in LB medium, present similar mechanisms of resistance to fosfomycin, the inactivation of enzymes belonging to the central carbon metabolism, while the resistance mechanism in clinical settings, urine and SCFM, appears to be not solely related to a metabolic shift. In urine, the resistance mechanism appears to be multifactorial and related to changes in central metabolism activities, whereas in the most relevant environment in infections caused by S. maltophilia, SCFM, the cause of resistance is not related, at least in the first instance, to changes in the central metabolism. 
A

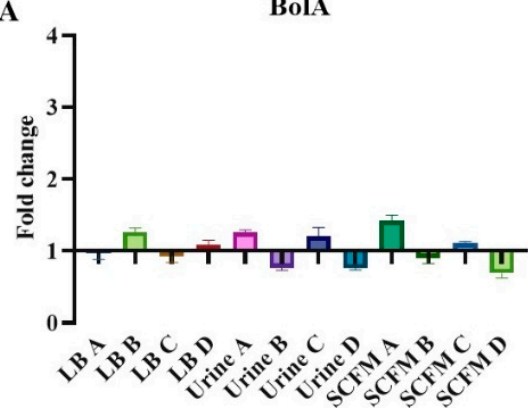

C

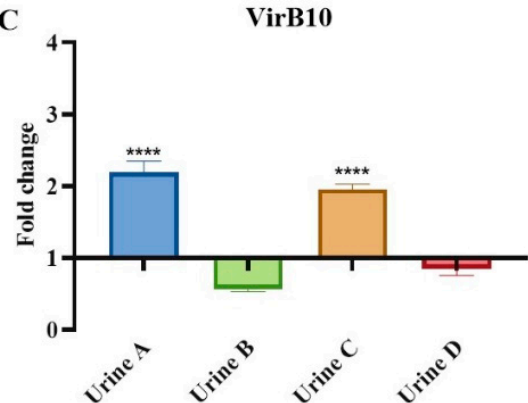

E

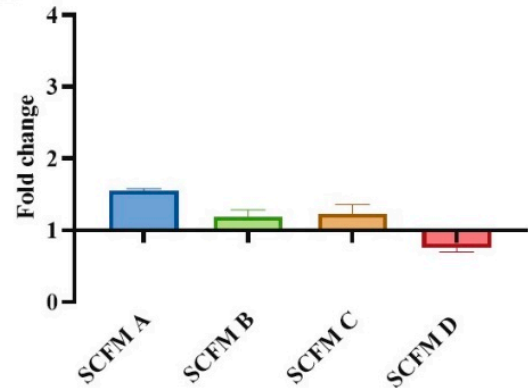

G

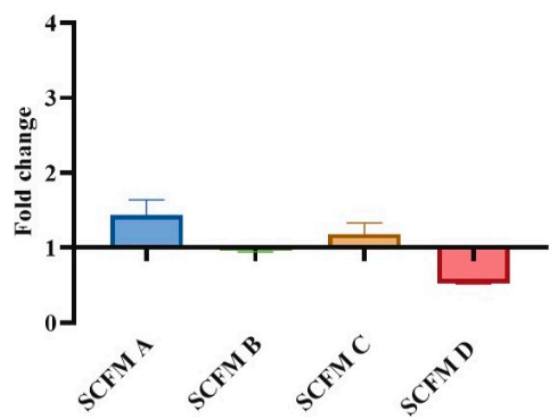

B
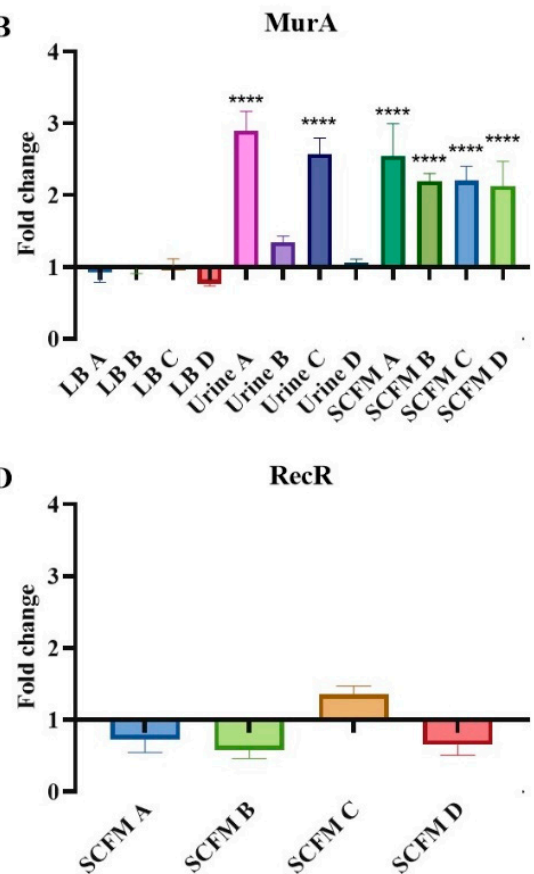

F

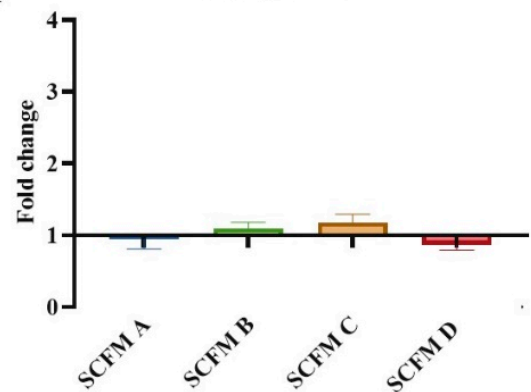

Figure 3. Effect of main mutations in gene expression. Expression level of genes encoding mutated genes. Fold changes in fosfomycin-evolved population were estimated regarding the expression of the D457 wild-type strain by qRT-PCR. (A) bolA expression; (B) murA expression; (C) virB10 expression; (D) recR expression; (E) $g s p L$ expression; (F) SMD_RS14605 expression; (G) $p t s N$ expression. Error bars indicate standard deviations of the results from three biological replicates. Statistically significant differences regarding D457 were calculated with t-test for paired samples assuming equal variances: **** $p<0.0001$. 


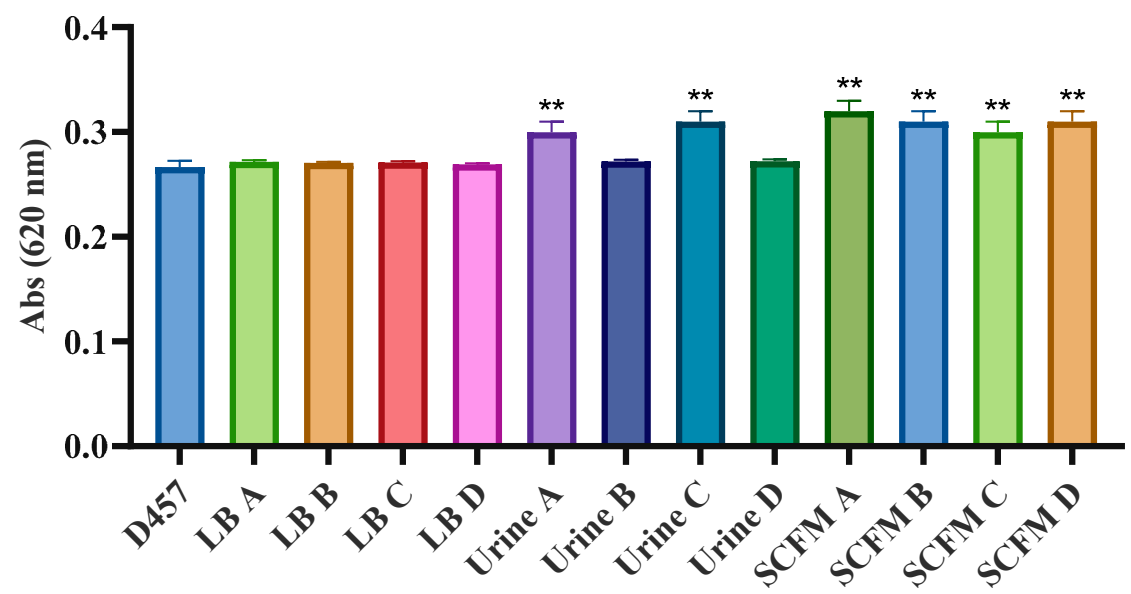

Figure 4. In vitro MurA activity measurements. Whole-cell lysates were preincubated with $10 \mathrm{mM}$ UDP-GlcNAc, $2 \mathrm{mM}$ DTT, and $50 \mathrm{mM}$ Tris. To each sample, $10 \mathrm{mM}$ PEP was added to start the reaction. Release of inorganic phosphate was measured by measuring the $\mathrm{OD}_{620}$ of the sample. Inorganic phosphate release was measured in triplicate. Statistically significant differences regarding D457 were calculated with t-test for paired samples assuming equal variances: ${ }^{* *} p<0.005$.

\subsection{Fosfomycin Resistance Is not Related to an Impaired Entry of Fosfomycin Inside the Cell}

Despite the fact that the S. maltophilia genome does not harbor genes encoding either the canonical fosfomycin transporters or already known fosfomycin-inactivating enzymes, it might be possible that other elements may contribute to impairing the accumulation of the antibiotic inside the studied populations. Additionally, the only common crossresistance change among most populations is the resistance to colistin, an antibiotic that diffuses through membrane. It may be also possible that mutations in the secretion system, observed in urine and SCFM-evolved populations, produce changes in the permeability of the outer membrane.

To analyze the possibility of an impaired antibiotic transport, the intracellular accumulation of fosfomycin in the different populations was measured. In any fosfomycin-evolved populations, the amount of intracellular fosfomycin was lower (Figure 7). These results confirm that the fosfomycin resistance is not due to a reduced intracellular concentration of this antibiotic in any of the fosfomycin-evolved populations. Further, an increased intracellular fosfomycin concentration was observed in some populations, being statistically significant in SCFM A- and B-evolved populations. These populations share an SNP in the gene $g s p L$, which is not mutated in any other population. This gene encodes a fimbrial assembly protein, an inner membrane component of the T2SS that provides a link between the energy-providing GspE protein in the cytoplasm and the rest of the T2SS machinery [24]. This mutation in the secretion systems could change outer membrane permeability leading to an enhanced uptake of molecules across the outer membrane into the periplasm. In addition, the SNP observed in SMD_RS14605 in lineage SCFM A could be also related to an increased intracellular fosfomycin concentration. Despite this increase in the intracellular concentration of fosfomycin, both populations show resistance to this antibiotic, which suggests that other resistance mechanisms, such as the higher concentration and activity of MurA (see above), are capable of producing resistance despite the increase in the intracellular concentration of fosfomycin. 
A

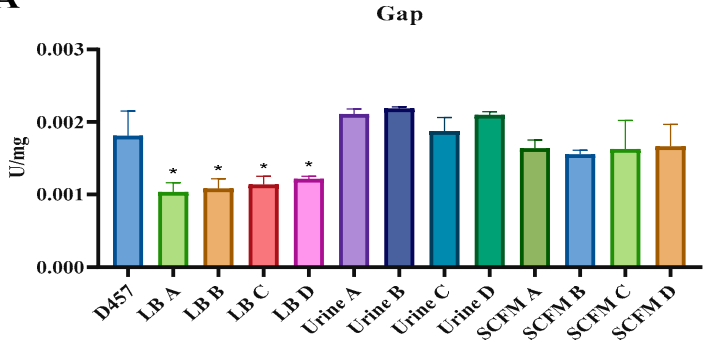

C

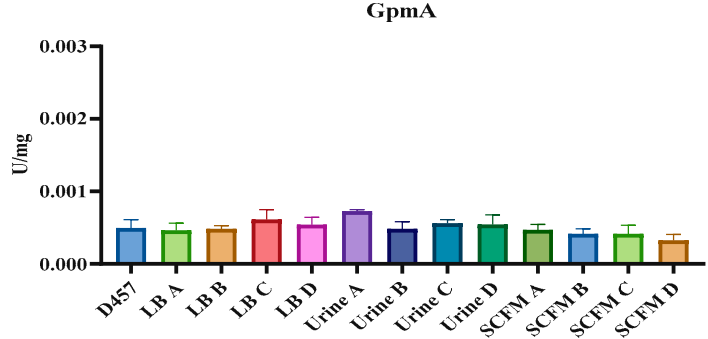

B

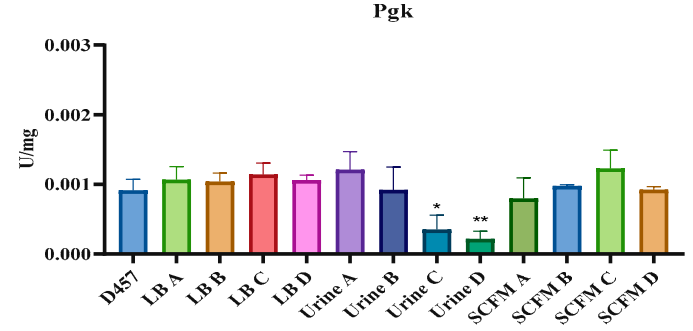

D

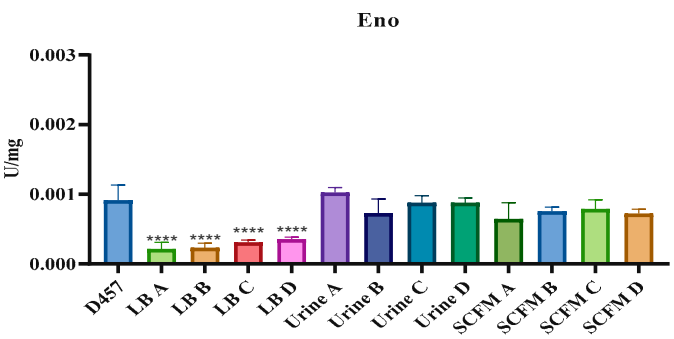

F

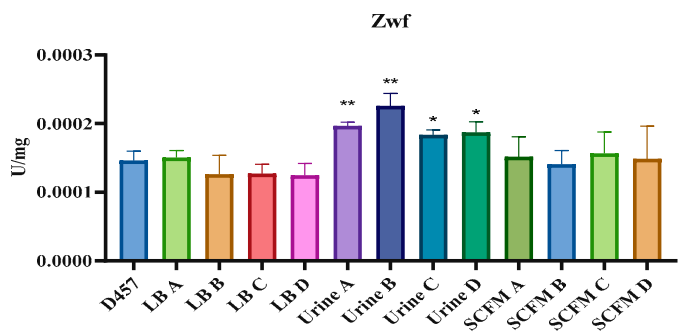

Figure 5. Enzymatic activity of the main enzymes of the glycolytic pathway of the D457 parental strain and the fosfomycin-evolved populations. (A) Zwf, glucose-6-phosphate dehydrogenase activity. (B) Gap, glyceraldehyde-3-phosphate dehydrogenase activity. (C) Pgk, phosphoglycerate kinase activity. (D) Gpm, phosphoglycerate mutase activity. (E) Eno, enolase activity. (F) Pyk, pyruvate kinase. Error bars indicate standard deviations for the results from three independent replicates. Values that are significantly different from the value for the wild-type D457 strain by an unpaired two-tail t-test are indicated by asterisks as follows: ${ }^{*}, p<0.05 ;{ }^{* *}, p<0.005 ;{ }^{* * *}, p<0.0001$. 


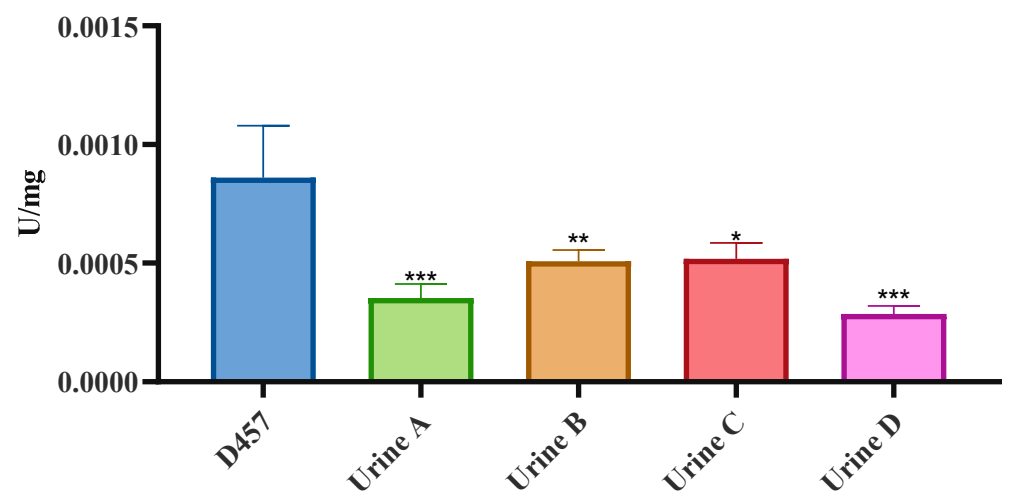

Figure 6. Enolase enzymatic activity of the D457 parental strain and isolated clones from the fosfomycin-evolved S. maltophilia populations in urine. Error bars indicate standard deviations for the results from three independent replicates. Values that are significantly different from the value for the wild-type D457 strain by an unpaired two-tail t-test are indicated by asterisks as follows: ${ }^{*}, p<0.05 ;{ }^{* *}, p<0.005 ;{ }^{* * *}, p<0.0005$.
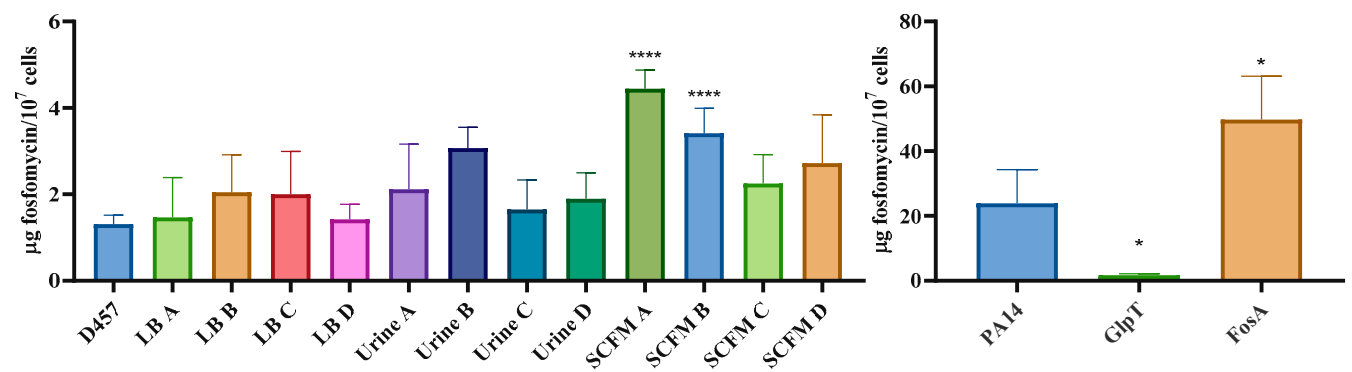

Figure 7. Fosfomycin-evolved populations' intracellular concentration of fosfomycin. Comparison of the fosfomycin intracellular concentration between the fosfomycin-evolved populations and the parental strain. There is not a deficiency in the fosfomycin transport in the populations that determines its resistance, or a fosfomycin-modifying enzyme involved in this resistance. PA14 and its mutants GlpT and FosA were used as controls for the assay. The amount of intracellular fosfomycin was lower in P. aeruginosa when its fosfomycin transporter GlpT was inactivated. An increased fosfomycin concentration was observed in the FosA mutant relative to the parental PA14 strain. Error bars indicate standard deviations for the results from three independent replicates. Statistical significance was calculated by unpaired two-tail t-test: ${ }^{*}, p<0.05 ;{ }^{* * *}, p<0.0001$.

\section{Discussion}

Although it has been described that fosfomycin resistance is associated with a high biological cost in the absence of fosfomycin, which may lead to the low prevalence of fosfomycin-resistant strains [44], using in vitro experimental evolution we have determined the first adaptive events driven by the exposure of $S$. maltophilia to increasing concentrations of fosfomycin in different settings, both laboratory and clinical. The evolutionary changes leading to fosfomycin resistance have been shown to be different in each condition and similar in the four parallel cultures of each medium. We have proved that even though evolutionary trajectories are different depending on the medium in which bacteria are subjected to a selective pressure, all selected mutations are able to cause resistance in all tested media. Moreover, as far as our knowledge reaches, none of the mutations have been associated with fosfomycin resistance in other bacterial pathogens besides S. maltophilia.

Fosfomycin resistance mechanisms described so far include the three classical categoriesalterations in fosfomycin transport, antibiotic inactivation and alterations in the target enzyme or peptidoglycan biosynthesis $[17,45]$ —along with the recently described inactivation of enzymes belonging to the EMP pathway. This last category was described after the study of one-step laboratory-isolated mutants obtained on LB agar medium with a supra-inhibitory fosfomycin 
concentration [19]. Our results show that experimental evolutions in LB medium result in mutations in one of the previously described enzymes, the inactivation of which leads to fosfomycin resistance: the enzyme enolase from the lower-glycolytic metabolic pathway. This mutation impairs the enolase activity, together with a decrease in the activity of the glyceraldehyde3-phosphate dehydrogenase and the pyruvate kinase lower-glycolysis enzymes. All these facts strongly suggest that mutations of genes encoding metabolic enzymes are the cause of $S$. maltophilia acquiring fosfomycin resistance in laboratory media.

Our study enables us to find different evolutionary trajectories depending on the medium in which the selection is carried out; due to the fact that no possible fosfomycin transporter has been found in the S. maltophilia genome, it allows finding alternative routes to the mutation of the canonical transporters of this antibiotic as the first acquired mechanism of resistance [46]. WGS analysis has indicated that mutations in genes encoding lower-glycolysis enzymes are also on the basis of the acquisition of resistance in urine. Despite the fact that eno mutations are found in a small proportion inside the populations without changing enolase activity at the population level, urine-evolved populations show a different physiological state as evidenced by an increased Zwf activity, an enzyme that supplies the cofactor NADPH needed to maintain cellular redox balance [47,48]. Together with this mutation, all urine-evolved populations present a one-pair insertion in the virB10-encoding gene. Even though mutations in this gene have been previously related to an increase in antibiotic susceptibility [23], and the present mutation seems to be deleterious for the gene, the results strongly suggest that mutations in T4SS are related to fosfomycin resistance in urine, since this mutation is present in all four linages. Furthermore, bolA mutation may be related to an increased synthesis and activity of the only known fosfomycin target, the enzyme MurA. Additionally, BolA homologs have been previously shown to be involved in acid and cell envelope stressors resistance at the same time that their deletions lead to reduced peptidoglycan and altered outer membrane lipids, leading to fosfomycin and vancomycin susceptibility $[40,41]$. Regardless, the mutation found is not deleterious to BolA, which leads us to think that its participation in S. maltophilia fosfomycin resistance is related to an increase in the expression of MurA; a fact that should be studied in more detail.

Previous analyses have shown that fosfomycin activity is higher in urine under acidic conditions [49]. In acidic urine ( $\mathrm{pH} 5$ to 6), fosfomycin is partially protonated and enters bacteria, resulting in higher fosfomycin activity. Therefore, urine acidification increases fosfomycin susceptibility even in the case of resistance isolates, including high-level fosfomycin-resistant mutant strains. At $\mathrm{pH} 5$ most strains are susceptible to fosfomycin. In UTIs caused by E. coli, urine is mainly acidic, with $\mathrm{pH}$ levels lower than 6.5, a fact that could explain the low prevalence of fosfomycin-resistant E. coli in these infections [49]. We detected an increased alkalization in urine from a $\mathrm{pH}$ value of 6-7 to 8 after $24 \mathrm{~h}$ of $S$. maltophilia growth. This alkaline $\mathrm{pH}$ might compromise fosfomycin activity and would allow the selection of mutations conferring low-level fosfomycin resistance. Despite this possibility, our results illustrate that urine-evolved populations are fosfomycin resistant in all tested media apart from urine. It is clear that $S$. maltophilia-selected mutations in urine award high-level fosfomycin resistance. However, it is also worth stating that, as above mentioned, UTIs caused by this microorganism have been rarely reported, a feature that somehow might reduce the clinical impact of our findings.

Moving to the most relevant clinical environment where S. maltophilia infections may occur, cystic fibrosis, first acquired fosfomycin resistance in this SCFM is definitely not related to a general metabolic shift. All four lineages present two common mutations: an SNP in bolA that has also been found in urine and an insertion upstream of recR. Since four SCFM and two urine lineages present a bolA mutation, it is clear that this not-deleterious mutation is the basis of fosfomycin-acquired resistance in clinically relevant media. In addition, these populations show an increased synthesis and expression of MurA, leading to fosfomycin resistance. 
Apart from these common mutations, SCFM lineages A and B present extra mutations that do not significantly increase the level of fosfomycin resistance compared with the other two lineages. Firstly, a single-nucleotide variant in $g s p L$ was found in both A and B lineages. This protein plays a role in the complex assembly and recruits GspM, resulting in a stable complex in the inner membrane [24]. Mutations in secretion system genes could lead to permeability changes in the outer membrane, as has been previously mentioned, a fact that explains why these two populations present a higher intracellular fosfomycin concentration than their parental strain. This kind of mutation enhances sensitivity to molecules that normally inhibit cell wall synthesis and do not enter the cell through porins, being consistent with this last result. Despite this fact, these two populations present a fosfomycin resistance phenotype and do not show collateral sensitivity to antibiotics such as vancomycin. Secondly, population A presents a SNP in SMD_RS14605 gene and another in $p t s N$ gene, both related to transport across the membrane. In addition, SMD_RS14605 is homologous to the $m s b A$ gene of $P$. aeruginosa, which has been previously related to antibiotic susceptibility.

MsbA has been suggested to be involved in active efflux, as well as in LPS production, carrying out the first essential step in the trafficking of LPS to the outer membrane [50]. Thus, its deficiency decreases LPS production, increasing outer membrane permeability, and consequently enhances membrane permeability for the toxic substrates moving in, including hydrophobic antibiotics that are not pumped out by MsbA [31]. All these facts suggest that a deleterious mutation of the gene encoding this $A B C$ inner transport protein would increase susceptibility to antibiotics such as aminoglycosides or chloramphenicol [51-55]; a fact that we did not observe. In addition, its deficiency would increase the intracellular concentration of these antibiotics it pumps out, suggesting that fosfomycin would be one of these antibiotics even though the increased intracellular fosfomycin concentration does not result in an associated increased susceptibility.

The acquisition of all these mutations may imply changes in susceptibility to other antibiotics apart from fosfomycin. To test this possibility, the susceptibility to other antibiotics belonging to different families was assessed in all the fosfomycin-evolved populations. Not many changes were observed, suggesting that the selected mutations specifically confer resistance to fosfomycin. However, two important changes attract attention: cross-resistance to colistin, which could suggest a membrane deficiency and collateral susceptibility to SXT, the most used antibiotic for treating S. maltophilia infections [6]. This last change suggests that the use of fosfomycin in combination with SXT could be a useful alternative for treating SXT-resistant S. maltophilia infections. These results show that the different media result in similar outcomes regarding susceptibility to other antibiotics not used in selection.

One important factor influencing the emergence of antibiotic-resistant bacteria is the resistance-associated fitness cost. Even though the acquisition of resistance implies a fitness advantage in the presence of the antibiotic of selection, in the absence of the selection, pressure-resistant bacteria would be outcompeted by susceptible ones [56,57]. Our data show that in the absence of the antibiotic of selection, all evolved populations present a fitness cost in LB and SCFM media. However, when tested in urine, even though LB- and SCFM-evolved populations displayed a fitness cost, urine-evolved populations presented a greater growth than the wild-type strain. All in all, in the absence of antibiotics it could be difficult for most fosfomycin-resistant mutants to be maintained in the population [58].

Our results once again show the importance of the interlink between the modification of the activity of enzymes belonging to central metabolism and the antibiotic susceptibility, which should be added as a new molecular mechanism of fosfomycin resistance, together with those traditionally described. In addition, we have been able to find fosfomycin resistance adaptive pathways that do not include permeability changes as the initial step due to a mutation in the genes encoding canonical fosfomycin transporters. In the same way, our results prove that the acquisition of resistance is different depending on the medium used, obtaining different main resistance mechanisms depending on the medium in which bacteria are subjected to the antibiotic pressure. Our results then highlight the 
need to use conditions that mimic actual bacterial infections in the search for resistance mutational trajectories by experimental evolution.

\section{Materials and Methods}

\subsection{Growth Conditions and Antibiotic Susceptibility Assays}

Bacteria were grown using liquid LB (lysogeny broth) Lennox medium, sterile urine from 6 healthy people ( 3 men and 3 women) that were between 25 and 55 years old and had not received antibiotics in the year before, or SCFM [19] at $37{ }^{\circ} \mathrm{C}$ with constant agitation at $250 \mathrm{rpm}$. Evolution experiments were performed with S. maltophilia D457, a model strain that had been isolated from a bronchial aspirate [59]. Fosfomycin (Sigma) was used at different concentrations during the evolution period. Antibiotic susceptibility testing was carried out in both the wild-type strain S. maltophilia D457 and all the fosfomycin-evolved populations. The MICs of fosfomycin and vancomycin were determined by double dilution in 96-well microtiter plates (NunclonTM Delta Surface) in LB at $37^{\circ} \mathrm{C}$. MICs of amikacin, streptomycin, tetracycline, tigecycline, ciprofloxacin, ofloxacin, aztreonam, nalidixic acid, gentamicin, ceftazidime, chloramphenicol, trimethoprim/sulfamethoxazole, colistin and polymyxin B were determined using MIC test strips (Liofilchem) on Mueller-Hinton (MH) agar plates at $37^{\circ} \mathrm{C}$ for $20 \mathrm{~h}$. Overnight bacterial cultures were normalized to an $\mathrm{OD}_{600}$ of 2, and a 1:1000 dilution of each culture was inoculated in the test plates.

\subsection{Experimental Evolutions}

Experimental evolutions were performed with the wild-type D457 strain growing in LB medium, urine or SCFM in the presence of increasing concentrations of fosfomycin. Eight independent experimental evolutions in each medium (four in the presence of fosfomycin and four controls without antibiotic) were carried out over a period of 3 days. Fosfomycin-challenged S. maltophilia D457 populations were initially grown at the maximum concentration that allowed bacterial growth in these conditions $(128 \mu \mathrm{g} / \mathrm{mL}$ in LB, $256 \mu \mathrm{g} / \mathrm{mL}$ in urine and $512 \mu \mathrm{g} / \mathrm{mL}$ in SCFM). Five hundred microliters of a bacterial overnight culture were inoculated in flasks containing $20 \mathrm{~mL}$ of the corresponding medium. Serial passages were performed every $24 \mathrm{~h}$ inoculating $500 \mu \mathrm{L}$ of bacterial cell cultures in fresh medium containing twice the concentration of fosfomycin, until fosfomycin concentration increased 4-fold. Controls were also cultured in the same conditions but in the absence of antibiotic. Samples were preserved at $-80^{\circ} \mathrm{C}$ every $24 \mathrm{~h}$.

\subsection{DNA Extraction, WGS and Identification of Mutations}

Total genomic DNA from the evolved populations and the wild-type D457 strain were extracted using a Gnome ${ }^{\circledR}$ DNA kit following the manufacturer's protocol (MP Biomedicals). Quality and quantity of the extracted DNA was determined by agarose gel electrophoresis and using a NanoDrop Spectrophotometer, respectively. WGS was performed by Macrogen Inc. using the HiSeq 2000 Sequencing System (Illumina) generating $150 \mathrm{bp}$ paired-end reads. The number of reads per sample was 5,000,000 on average, which represents a sequencing coverage of approximately $100 \times$. Illumina short reads $(2 \times 150 \mathrm{bp})$ were checked with FASTQC (Simon Andrews's group. https: / /www. bioinformatics.babraham.ac.uk/projects/fastqc/ Last access date: 15 December 2021). The S. maltophilia D457 reference genome (NC_017671.1) was used to align the WGS obtained using RNA STAR [60] (-alignIntronMax = 1). SNPs and small insertions/deletions (INDEL) were detected using freebayes (-strict-vcf -pooled-continuous-P 1). Only primary alignments, not marked as duplicated, were considered ('effective reads'). Impact of detected SNP and INDEL was evaluated using snpEff [61]. Genomic regions with no coverage (large deletions) were detected using bedtools genomecov command [62]. The given variants were filtered against those obtained for the wild-type D457 strain and visualized by SNPer (https:/ / bioinfogp.cnb.csic.es/tools/snper_dev/ Last access date: 15 December 2021). The presence of each putative mutation was verified by polymerase chain reaction (PCR) using the corresponding pairs of primers (Table S1). DNA fragments were purified using the 
QIAquick PCR Purification kit (QIAGEN, Hilden, Germany) and Sanger sequencing was performed at GATC Biotech.

\subsection{Fitness Cost Determination}

Bacterial samples from the fosfomycin-evolved populations were used for the assay. A $10 \mu \mathrm{L}$ sample of each culture was inoculated in $140 \mu \mathrm{L}$ of LB medium, urine or SCFM to a final $\mathrm{OD}_{600}$ of 0.01 using 96-well plates (Nunc MicroWell; Thermo Fisher; Waltham, $\mathrm{MA}, \mathrm{USA})$. Growth $\left(\mathrm{OD}_{600}\right)$ from three independent replicates was monitored every $10 \mathrm{~min}$ using a Spark $10 \mathrm{M}$ plate reader (Tecan; Männedorf, Switzerland) for $24 \mathrm{~h}$ at $37^{\circ} \mathrm{C}$. Shaking for $10 \mathrm{~s}$ was performed before each measurement. $\mathrm{OD}_{600}$ values at the exponential growth phase were used to calculate the area under the curve. Relative growth rates were calculated by dividing the values of area under the curve of each population by those obtained for the parental strain D457 from the same experiment.

\subsection{RNA Extraction and Quantitative Reverse Transcription PCR ( $q R T-P C R)$}

S. maltophilia D457 wild-type and all the evolved populations were grown overnight in LB broth at $37^{\circ} \mathrm{C}$ and $250 \mathrm{rpm}$. These cultures were used to inoculate new flasks to reach an optical density at $600 \mathrm{~nm}\left(\mathrm{OD}_{600}\right)$ of 0.01 , and the cultures were grown at $37^{\circ} \mathrm{C}$ until an $\mathrm{OD}_{600}$ of 0.6 was reached. RNA was isolated following the protocol described by Gil-Gil T et al. [19]. DNA contamination was checked by PCR with primers 27 and 48 (Table S1). Only RNAs containing no DNA contamination were used for further studies. Using a high-capacity cDNA reverse transcription kit (Applied Biosystems, Waltham, MA, USA), cDNA was obtained from $10 \mu \mathrm{g}$ of RNA. qRT-PCR was carried out with a Power SYBR green PCR master mix (Applied Biosystems) in an ABI Prism 7500 real-time system (Applied Biosystems). A total of $50 \mathrm{ng}$ of cDNA was used in each reaction, except for the wells with no template used as negative controls. A first denaturation step at $95{ }^{\circ} \mathrm{C}$ for $10 \mathrm{~min}$ was followed by 40 cycles at $95^{\circ} \mathrm{C}$ for $15 \mathrm{~s}$ and $60^{\circ} \mathrm{C}$ for $1 \mathrm{~min}$ for amplification and quantification. Primers that amplify specific fragments of the desired genes were designed with Primer3 Input software (v. 0.4.0) and were used at $400 \mathrm{nM}$ (Table S1). The primers gyrA_F and gyrA_R were used to amplify the housekeeping gene gyrA (Table S1). Differences in the relative amounts of mRNA were determined according to the $2^{-\Delta \Delta C T}$ method [63]. In all cases, the values of relative mRNA expression were determined as the average of three independent biological replicates, each containing two technical replicates.

\subsection{Assay of MurA Activity}

Cells were grown until exponential phase $\left(\mathrm{OD}_{600}=0.6\right)$ and harvested by centrifugation at $5100 \times \mathrm{g}$ and $4{ }^{\circ} \mathrm{C}$ and washed twice in $0.9 \% \mathrm{NaCl}$ and $10 \mathrm{mM} \mathrm{MgSO} 4$. Once washed, cells were resuspended in $1 \mathrm{~mL}$ of $100 \mathrm{mM}$ Tris [pH 7.5]-5 mM MgCl2-1 mM dithiothreitol (DTT), disrupted by sonication at $4{ }^{\circ} \mathrm{C}$, and the cell extracts were obtained by centrifugation at $23,100 \times \mathrm{g}$ for $30 \mathrm{~min}$ at $4{ }^{\circ} \mathrm{C}$. Protein concentration was determined following the Pierce BCA Protein assay kit (Thermo Scientific; Waltham, MA, USA) protocol in 96-well plates (Nunc MicroWell; Thermo Fisher; Waltham, MA, USA).

The assay mixture (final volume, $72 \mu \mathrm{L}$ ) contained $50 \mathrm{mM}$ Tris at $\mathrm{pH}$ 7.5, $2 \mathrm{mM}$ DTT and $10 \mathrm{mM}$ UDP-GlcNAc, and $200 \mu \mathrm{g} / \mathrm{mL}$ of bacterial extract protein. Samples were preincubated for $15 \mathrm{~min}$ at $37^{\circ} \mathrm{C}$, and the reaction was started by the addition of $8 \mu \mathrm{L}$ of $10 \mathrm{mM}$ PEP. After $2 \mathrm{~h}$ of incubation at $37^{\circ} \mathrm{C}, 20 \mu \mathrm{L}$ of color reagent (bicinchoninic acid solution and Copper (II) sulfate solution) was added to stop the reaction and measure the release of orthophosphate $(\mathrm{Pi})$, monitored every $30 \mathrm{~min}$ using a Spark $10 \mathrm{M}$ plate reader (Tecan) for $2 \mathrm{~h}$. Results are expressed as $\mathrm{OD}_{620}$ values corrected for the background reading in the absence of UDP-GlcNAc.

\subsection{In Vitro Activity Assays of the Enzymes of the Lower Glycolytic Pathway and Dehydrogenases}

Protein extracts and protein quantification were performed as above mentioned. $\mathrm{NAD}(\mathrm{P})+$ reduction or $\mathrm{NAD}(\mathrm{P}) \mathrm{H}$ oxidation was monitored spectrophotometrically at 
$340 \mathrm{~nm}$ and $25^{\circ} \mathrm{C}$ with intermittent shaking in microtiter plates using a Spark $10 \mathrm{M}$ plate reader (Tecan; Männedorf, Switzerland). Each reaction was performed using three biological replicates, and the specific activities were obtained by dividing the measured slope of $\mathrm{NAD}(\mathrm{P}) \mathrm{H}$ formation or consumption by the total protein concentration. Enzymatic activities of dehydrogenases (Zwf and Gap) were measured as described previously [19]. Enzymatic activities of Pgk, Pgm, Eno and Pyk were assayed following the protocol described by Gil-Gil, T et al. [19] in a two-step reaction.

\subsection{Quantification of Intracellular Fosfomycin}

Assays to test fosfomycin accumulation in bacterial cells were conducted as previously stated [19]. For measuring the amount of intracellular concentration of fosfomycin, fosfomycin-evolved populations, S. maltophilia D457 wild-type strain and P. aeruginosa PA14 wild-type strain, as well as $P$. aeruginosa $\Delta g l p T$ and $\Delta f o s A$ as controls, were used. The fosfomycin concentration is represented as micrograms per $10^{7}$ cells.

Supplementary Materials: The following are available online at https: / www.mdpi.com/article / 10.3390 /ijms23031132/s1.

Author Contributions: Conceptualization, review and editing: T.G.-G. and J.L.M.; methodology, investigation and original draft: T.G.-G.; supervision, project administration and funding acquisition: J.L.M. All authors have read and agreed to the published version of the manuscript.

Funding: This work was supported by Instituto de Salud Carlos III (grant RD16/0016/0011)cofinanced by the European Development Regional Fund "A Way to Achieve Europe", by grant S2017/BMD-3691 InGEMICS-CM, funded by Comunidad de Madrid (Spain) and European Structural and Investment Funds and by MCIN/AEI /10.13039/501100011033 (PID2020-113521RB-I00). T.G.G. is the recipient of a FPI fellowship from MINECO.

Institutional Review Board Statement: The work does not include studies involving humans or other animals.

Informed Consent Statement: Informed consent was obtained from all subjects involved in the study.

Data Availability Statement: All data used in the work have been included in the manuscript.

Conflicts of Interest: The authors declare no conflict of interest.

\section{References}

1. Kaye, K.S.; Pogue, J.M. Infections Caused by Resistant Gram-Negative Bacteria: Epidemiology and Management. Pharmacotherapy 2015, 35, 949-962. [CrossRef]

2. Behera, B.; Mohanty, S.; Sahu, S.; Praharaj, A.K. In vitro Activity of Fosfomycin against Multidrug-Resistant Urinary and Nonurinary Gram-Negative Isolates. Indian J. Crit. Care Med. 2018, 22, 533-536. [PubMed]

3. Brooke, J.S.; Di Bonaventura, G.; Berg, G.; Martinez, J.L. Editorial: A Multidisciplinary Look at Stenotrophomonas maltophilia: An Emerging Multi-Drug-Resistant Global Opportunistic Pathogen. Front. Microbiol. 2017, 8, 1511. [CrossRef] [PubMed]

4. Brooke, J.S. Stenotrophomonas maltophilia: An emerging global opportunistic pathogen. Clin. Microbiol. Rev. 2012, 25, 2-41. [CrossRef] [PubMed]

5. Vartivarian, S.E.; Papadakis, K.A.; Anaissie, E.J. Stenotrophomonas (Xanthomonas) maltophilia urinary tract infection. A disease that is usually severe and complicated. Arch. Intern. Med. 1996, 156, 433-435. [CrossRef] [PubMed]

6. Gil-Gil, T.; Martínez, J.L.; Blanco, P. Mechanisms of antimicrobial resistance in Stenotrophomonas maltophilia: A review of current knowledge. Expert Rev. Anti-Infect. Ther. 2020, 18, 335-347. [CrossRef] [PubMed]

7. Green, H.; Jones, A.M. The microbiome and emerging pathogens in cystic fibrosis and non-cystic fibrosis bronchiectasis. Semin. Respir. Crit. Care Med. 2015, 36, 225-235. [CrossRef]

8. Esposito, A.; Pompilio, A.; Bettua, C.; Crocetta, V.; Giacobazzi, E.; Fiscarelli, E.; Jousson, O.; Di Bonaventura, G. Evolution of Stenotrophomonas maltophilia in Cystic Fibrosis Lung over Chronic Infection: A Genomic and Phenotypic Population Study. Front. Microbiol. 2017, 8, 1590. [CrossRef]

9. Pompilio, A.; Crocetta, V.; Ghosh, D.; Chakrabarti, M.; Gherardi, G.; Vitali, L.A.; Fiscarelli, E.; Di Bonaventura, G. Stenotrophomonas maltophilia Phenotypic and Genotypic Diversity during a 10-year Colonization in the Lungs of a Cystic Fibrosis Patient. Front. Microbiol. 2016, 7, 1551. [CrossRef]

10. Falagas, M.E.; Giannopoulou, K.P.; Kokolakis, G.N.; Rafailidis, P.I. Fosfomycin: Use beyond urinary tract and gastrointestinal infections. Clin. Infect. Dis. 2008, 46, 1069-1077. [CrossRef] 
11. European Association of Urology. European Association of Urology Guidelines. In EAU Annual Congress, Milan 2021; 2021; ISBN 978-94-92671-13-4.

12. Raz, R. Fosfomycin: An old-New antibiotic. Clin. Microbiol. Infect. 2012, 18, 4-7. [CrossRef] [PubMed]

13. Allen, R.C.; Pfrunder-Cardozo, K.R.; Hall, A.R. Collateral Sensitivity Interactions between Antibiotics Depend on Local Abiotic Conditions. mSystems 2021, 6, e0105521. [CrossRef] [PubMed]

14. Scribner, M.R.; Santos-Lopez, A.; Marshall, C.W.; Deitrick, C.; Cooper, V.S. Parallel Evolution of Tobramycin Resistance across Species and Environments. mBio 2020, 11, e00932-20. [CrossRef] [PubMed]

15. Kim, D.H.; Lees, W.J.; Kempsell, K.E.; Lane, W.S.; Duncan, K.; Walsh, C.T. Characterization of a Cys115 to Asp substitution in the Escherichia coli cell wall biosynthetic enzyme UDP-GlcNAc enolpyruvyl transferase (MurA) that confers resistance to inactivation by the antibiotic fosfomycin. Biochemistry 1996, 35, 4923-4928. [CrossRef]

16. Tsuruoka, T.; Yamada, Y. Characterization of spontaneous fosfomycin (phosphonomycin)-resistant cells of Escherichia coli B in vitro. J. Antibiot. 1975, 28, 906-911. [CrossRef] [PubMed]

17. Blair, J.M.; Webber, M.A.; Baylay, A.J.; Ogbolu, D.O.; Piddock, L.J. Molecular mechanisms of antibiotic resistance. Nat. Rev. Microbiol. 2015, 13, 42-51. [CrossRef] [PubMed]

18. Arca, P.; Hardisson, C.; Suárez, J.E. Purification of a glutathione S-transferase that mediates fosfomycin resistance in bacteria. Antimicrob. Agents Chemother. 1990, 34, 844-848. [CrossRef]

19. Gil-Gil, T.; Corona, F.; Martinez, J.L.; Bernardini, A. The Inactivation of Enzymes Belonging to the Central Carbon Metabolism Is a Novel Mechanism of Developing Antibiotic Resistance. mSystems 2020, 5, e00282-20. [CrossRef]

20. Gil-Gil, T.; Ochoa-Sánchez, L.E.; Martínez, J.L. The Antibiotic Fosfomycin Mimics the Effects of the Intermediate Metabolites Phosphoenolpyruvate and Glyceraldehyde-3-Phosphate on the Stenotrophomonas maltophilia Transcriptome. Int. J. Mol. Sci. 2021, 23, 159. [CrossRef]

21. Martinez, J.L.; Rojo, F. Metabolic regulation of antibiotic resistance. FEMS Microbiol. Rev. 2011, 35, 768-789. [CrossRef]

22. Bayer-Santos, E.; Cenens, W.; Matsuyama, B.Y.; Oka, G.U.; Di Sessa, G.; Mininel, I.D.V.; Alves, T.L.; Farah, C.S. The opportunistic pathogen Stenotrophomonas maltophilia utilizes a type IV secretion system for interbacterial killing. PLoS Pathog. 2019, 15, e1007651. [CrossRef]

23. Darbari, V.C.; Ciccone, J.; Patel, J.S.; Islam, B.; Agarwal, P.K.; Haider, S. Electrostatic Switching Controls Channel Dynamics of the Sensor Protein VirB10 in A. tumefaciens Type IV Secretion System. ACS Omega 2020, 5, 3271-3281. [CrossRef]

24. Karaba, S.M.; White, R.C.; Cianciotto, N.P. Stenotrophomonas maltophilia encodes a type II protein secretion system that promotes detrimental effects on lung epithelial cells. Infect. Immun. 2013, 81, 3210-3219. [CrossRef] [PubMed]

25. Zhou, Z.; White, K.A.; Polissi, A.; Georgopoulos, C.; Raetz, C.R. Function of Escherichia coli MsbA, an essential ABC family transporter, in lipid A and phospholipid biosynthesis. J. Biol. Chem. 1998, 273, 12466-12475. [CrossRef] [PubMed]

26. Doerrler, W.T.; Raetz, C.R. ATPase activity of the MsbA lipid flippase of Escherichia coli. J. Biol. Chem. 2002, 277, 36697-36705. [CrossRef]

27. Doerrler, W.T.; Gibbons, H.S.; Raetz, C.R. MsbA-dependent translocation of lipids across the inner membrane of Escherichia coli. J. Biol. Chem. 2004, 279, 45102-45109. [CrossRef] [PubMed]

28. Mi, W.; Li, Y.; Yoon, S.H.; Ernst, R.K.; Walz, T.; Liao, M. Structural basis of MsbA-mediated lipopolysaccharide transport. Nature 2017, 549, 233-237. [CrossRef]

29. Polissi, A.; Georgopoulos, C. Mutational analysis and properties of the msbA gene of Escherichia coli, coding for an essential ABC family transporter. Mol. Microbiol. 1996, 20, 1221-1233. [CrossRef] [PubMed]

30. Karow, M.; Georgopoulos, C. The essential Escherichia coli msbA gene, a multicopy suppressor of null mutations in the htrB gene, is related to the universally conserved family of ATP-dependent translocators. Mol. Microbiol. 1993, 7, 69-79. [CrossRef]

31. Chiu, H.C.; Lin, T.L.; Yang, J.C.; Wang, J.T. Synergistic effect of imp/ostA and msbA in hydrophobic drug resistance of Helicobacter pylori. BMC Microbiol. 2009, 9, 136. [CrossRef]

32. Jones, D.H.; Franklin, F.C.; Thomas, C.M. Molecular analysis of the operon which encodes the RNA polymerase sigma factor sigma 54 of Escherichia coli. Microbiology 1994, 140, 1035-1043. [CrossRef] [PubMed]

33. Powell, B.S.; Court, D.L.; Inada, T.; Nakamura, Y.; Michotey, V.; Cui, X.; Reizer, A.; Saier, M.H., Jr.; Reizer, J. Novel proteins of the phosphotransferase system encoded within the rpoN operon of Escherichia coli. Enzyme IIANtr affects growth on organic nitrogen and the conditional lethality of an erats mutant. J. Biol. Chem. 1995, 270, 4822-4839. [CrossRef]

34. Pfluger-Grau, K.; Gorke, B. Regulatory roles of the bacterial nitrogen-related phosphotransferase system. Trends Microbiol. 2010, 18, 205-214. [CrossRef] [PubMed]

35. Lee, C.R.; Park, Y.H.; Kim, M.; Kim, Y.R.; Park, S.; Peterkofsky, A.; Seok, Y.J. Reciprocal regulation of the autophosphorylation of enzyme INtr by glutamine and alpha-ketoglutarate in Escherichia coli. Mol. Microbiol. 2013, 88, 473-485. [CrossRef]

36. Lee, C.R.; Cho, S.H.; Yoon, M.J.; Peterkofsky, A.; Seok, Y.J. Escherichia coli enzyme IIANtr regulates the K+ transporter TrkA. Proc. Natl. Acad. Sci. USA 2007, 104, 4124-4129. [CrossRef] [PubMed]

37. Luttmann, D.; Heermann, R.; Zimmer, B.; Hillmann, A.; Rampp, I.S.; Jung, K.; Gorke, B. Stimulation of the potassium sensor KdpD kinase activity by interaction with the phosphotransferase protein IIA(Ntr) in Escherichia coli. Mol. Microbiol. 2009, 72, 978-994. [CrossRef] [PubMed]

38. Cases, I.; Lopez, J.A.; Albar, J.P.; De Lorenzo, V. Evidence of multiple regulatory functions for the PtsN (IIA(Ntr)) protein of Pseudomonas putida. J. Bacteriol. 2001, 183, 1032-1037. [CrossRef] 
39. Mohamed, Y.F.; Abou-Shleib, H.M.; Khalil, A.M.; El-Guink, N.M.; El-Nakeeb, M.A. Membrane permeabilization of colistin toward pan-drug resistant Gram-negative isolates. Braz. J. Microbiol. 2016, 47, 381-388. [CrossRef]

40. Guinote, I.B.; Moreira, R.N.; Freire, P.; Arraiano, C.M. Characterization of the BolA homolog IbaG: A new gene involved in acid resistance. J. Microbiol. Biotechnol. 2012, 22, 484-493. [CrossRef]

41. Fleurie, A.; Zoued, A.; Alvarez, L.; Hines, K.M.; Cava, F.; Xu, L.; Davis, B.M.; Waldor, M.K. A Vibrio cholerae BolA-Like Protein Is Required for Proper Cell Shape and Cell Envelope Integrity. mBio 2019, 10, e00790-19. [CrossRef]

42. Marquardt, J.L.; Siegele, D.A.; Kolter, R.; Walsh, C.T. Cloning and sequencing of Escherichia coli murZ and purification of its product, a UDP-N-acetylglucosamine enolpyruvyl transferase. J. Bacteriol. 1992, 174, 5748-5752. [CrossRef]

43. Kohanski, M.A.; Dwyer, D.J.; Hayete, B.; Lawrence, C.A.; Collins, J.J. A common mechanism of cellular death induced by bactericidal antibiotics. Cell 2007, 130,797-810. [CrossRef]

44. Nilsson, A.I.; Berg, O.G.; Aspevall, O.; Kahlmeter, G.; Andersson, D.I. Biological costs and mechanisms of fosfomycin resistance in Escherichia coli. Antimicrob. Agents Chemother. 2003, 47, 2850-2858. [CrossRef]

45. Falagas, M.E.; Vouloumanou, E.K.; Samonis, G.; Vardakas, K.Z. Fosfomycin. Clin. Microbiol. Rev. 2016, 29, 321-347. [CrossRef] [PubMed]

46. McCoy, A.J.; Sandlin, R.C.; Maurelli, A.T. In vitro and in vivo functional activity of Chlamydia MurA, a UDP-N-acetylglucosamine enolpyruvyl transferase involved in peptidoglycan synthesis and fosfomycin resistance. J. Bacteriol. 2003, 185, 1218-1228. [CrossRef] [PubMed]

47. Sandoval, J.M.; Arenas, F.A.; Vasquez, C.C. Glucose-6-phosphate dehydrogenase protects Escherichia coli from tellurite-mediated oxidative stress. PLoS ONE 2011, 6, e25573. [CrossRef]

48. Kim, J.; Jeon, C.O.; Park, W. Dual regulation of zwf-1 by both 2-keto-3-deoxy-6-phosphogluconate and oxidative stress in Pseudomonas putida. Microbiology 2008, 154, 3905-3916. [CrossRef]

49. Martin-Gutierrez, G.; Docobo-Perez, F.; Rodriguez-Beltran, J.; Rodriguez-Martinez, J.M.; Aznar, J.; Pascual, A.; Blazquez, J. Urinary Tract Conditions Affect Fosfomycin Activity against Escherichia coli Strains Harboring Chromosomal Mutations Involved in Fosfomycin Uptake. Antimicrob. Agents Chemother. 2018, 62. [CrossRef]

50. Alexander, M.K.; Miu, A.; Oh, A.; Reichelt, M.; Ho, H.; Chalouni, C.; Labadie, S.; Wang, L.; Liang, J.; Nickerson, N.N.; et al. Disrupting Gram-Negative Bacterial Outer Membrane Biosynthesis through Inhibition of the Lipopolysaccharide Transporter MsbA. Antimicrob. Agents Chemother. 2018, 62, e01142-18. [CrossRef] [PubMed]

51. Reyes, C.L.; Ward, A.; Yu, J.; Chang, G. The structures of MsbA: Insight into ABC transporter-mediated multidrug efflux. FEBS Lett. 2006, 580, 1042-1048. [CrossRef] [PubMed]

52. Leive, L.; Telesetsky, S.; Coleman, W.G., Jr.; Carr, D. Tetracyclines of various hydrophobicities as a probe for permeability of Escherichia coli outer membranes. Antimicrob. Agents Chemother. 1984, 25, 539-544. [CrossRef]

53. Vuorio, R.; Vaara, M. The lipid A biosynthesis mutation lpxA2 of Escherichia coli results in drastic antibiotic supersusceptibility. Antimicrob. Agents Chemother. 1992, 36, 826-829. [CrossRef]

54. Clements, J.M.; Coignard, F.; Johnson, I.; Chandler, S.; Palan, S.; Waller, A.; Wijkmans, J.; Hunter, M.G. Antibacterial activities and characterization of novel inhibitors of LpxC. Antimicrob. Agents Chemother. 2002, 46, 1793-1799. [CrossRef]

55. Reuter, G.; Janvilisri, T.; Venter, H.; Shahi, S.; Balakrishnan, L.; van Veen, H.W. The ATP binding cassette multidrug transporter LmrA and lipid transporter MsbA have overlapping substrate specificities. J. Biol. Chem. 2003, 278, 35193-35198. [CrossRef] [PubMed]

56. Andersson, D.I.; Hughes, D. Antibiotic resistance and its cost: Is it possible to reverse resistance? Nat. Rev. Microbiol. 2010, 8 , 260-271. [CrossRef] [PubMed]

57. Hernando-Amado, S.; Sanz-Garcia, F.; Blanco, P.; Martinez, J.L. Fitness costs associated with the acquisition of antibiotic resistance. Essays Biochem. 2017, 61, 37-48. [PubMed]

58. Levin, B.R.; Bull, J.J. Short-sighted evolution and the virulence of pathogenic microorganisms. Trends Microbiol. 1994, 2, 76-81. [CrossRef]

59. Sanchez, P.; Alonso, A.; Martinez, J.L. Regulatory regions of smeDEF in Stenotrophomonas maltophilia strains expressing different amounts of the multidrug efflux pump SmeDEF. Antimicrob. Agents Chemother. 2004, 48, 2274-2276. [CrossRef]

60. Dobin, A.; Davis, C.A.; Schlesinger, F.; Drenkow, J.; Zaleski, C.; Jha, S.; Batut, P.; Chaisson, M.; Gingeras, T.R. STAR: Ultrafast universal RNA-seq aligner. Bioinformatics 2013, 29, 15-21. [CrossRef]

61. Cingolani, P.; Platts, A.; Wang le, L.; Coon, M.; Nguyen, T.; Wang, L.; Land, S.J.; Lu, X.; Ruden, D.M. A program for annotating and predicting the effects of single nucleotide polymorphisms, SnpEff: SNPs in the genome of Drosophila melanogaster strain w1118; iso-2; iso-3. Fly 2012, 6, 80-92. [CrossRef]

62. Quinlan, A.R.; Hall, I.M. BEDTools: A flexible suite of utilities for comparing genomic features. Bioinformatics 2010, 26, 841-842. [CrossRef] [PubMed]

63. Livak, K.J.; Schmittgen, T.D. Analysis of relative gene expression data using real-time quantitative PCR and the 2(-Delta Delta C(T)) Method. Methods 2001, 25, 402-408. [CrossRef] [PubMed] 